\title{
Epidural blood patch - myths and legends
}

\author{
Mike Paech MBbS FRCA FANZCA FFPMANZCA DM
}

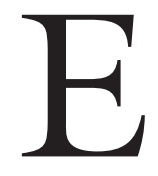

PIDURAL blood patch (EBP) was suggested before even I was born, and has been considered the most effective treatment of post-dural puncture for over 40 years, yet many aspects still fascinate and confuse, and its clinical merit remains controversial. A systematic review of EBP, in the highly respected Cochrane database of systematic reviews, concluded: " At present, except in the context of a randomised controlled trial, we believe that epidural blood patching should be reserved for exceptional cases only (such as) post-dural puncture headache complicated by subdural hemorrhage or disabling headache after one week or more."1 Wow, hands up if you agree!

EBP entered practice thanks to the work of a general surgeon, James Gormley, ${ }^{2}$ partly because of his serendipitous observation that bloody insertions were less likely to cause headache (although this is probably erroneous!). Knowledge of EBP was disseminated by the American anesthesiologist Anthony DiGiovanni, a legend in my eyes, not least because he, like many of us, had his manuscript rejected by Anesthesiology. The British legend was Selwyn Crawford, who gathered useful observational data and stimulated new research.

Given that rigorous investigation is difficult and noticeably lacking, it is not surprising that myths about EBP persist. Textbooks have tended to perpetuate a series of misconceptions about EBP after dural puncture by epidural needles, and data supporting a number of common practices are limited or of questionable validity, making evidence-based practice impossible.

To distinguish fact from fiction (or supposition at least), many aspects related to this therapy could be examined. Examples are questions such as: "Should blood culture be performed before EBP?"; and dictums such as: "Perform the EBP at the same or an interspace below the level of dural puncture", "Keep the patient supine for at least two hours after EBP" and: "Avoid straining after an EBP". Confining my comments principally to the obstetric population, I will attempt to identify the strength of evidence in relation to four areas of controversy or misconception:

1. The effectiveness of EBP after unintentional dural puncture with an epidural needle.

2. The volume of autologous blood that should be injected for EBP.

3. The optimal timing of EBP.

4. The safety of EBP.

5. The mechanism by which EBP relieves headache.

\section{"How effective is an EBP after an unintentional dural puncture?"}

Obstetric anesthesiologists working in busy units with trainees get to become "experts" because they are involved with, or hear about, a few EBP each year. This highlights that our individual exposure to this intervention, over a lifetime, is extremely limited (at least we hope!). Consequently, personal experience and anecdote tend to strongly colour our opinions and practices. The Cochrane review of randomized controlled trials of EBP vs no EBP for treatment of post-dural puncture headache (PDPH), between 1980 and 2000, found only three trials suitable for analysis (two on prophylactic EBP), all others being confounded, non-randomized or otherwise unreliable. ${ }^{1}$ Analysis showed an effect size favouring EBP, but the sample size of the studies was too small to be conclusive $(n=12$ in the only trial of therapeutic EBP). In addition, the latter trial included patients of mixed gender, almost half of whom suffered headache after puncture by a spinal rather than epidural needle, in some cases many months previously. So clearly data is inadequate, but I can't agree with the reviewers' provocative recommendation. As someone said recently, you don't need a randomized trial to decide whether it's worth using a parachute when you jump from a plane! What the Cochrane review highlights is how important it is that clinicians and investigators address this topic henceforth in a scientifically rigorous fashion.

From the School of Medicine and Pharmacology, University of Western Australia, Perth, Australia.

Address correspondence to: Dr. Mike Paech, Department of Anaesthesia and Pain Medicine, King Edward Memorial Hospital for Women, 374 Bagot Rd., Subiaco, WA 6008, Australia. Phone: +61 89340 2222; Fax: +61 89340 2260;

E-mail: michael.paech@health.wa.gov.au 
Many studies, textbooks and review articles fail to distinguish between the efficacy of EBP for PDPH secondary to small gauge spinal needle puncture and for PDPH after a 16-18 gauge epidural needle puncture. Therapeutic EBP clearly has much better success in relieving PDPH induced by the former $(95 \%$ or greater) than for the latter (30-75\%). ${ }^{3,4}$ Exaggerated quotes for success are also a consequence of outcomes found in older series in which bias, limited assessment and an inadequate duration of follow-up are apparent. Although initial relief from an EBP is high, many parturients experience only a partial reduction in the severity of PDPH and the frequency of recurrence or exacerbation is significant (about 30\%). ${ }^{5,6}$ Women should be informed that the chance of a "cure" (complete relief) with a single EBP is at best $50 \%$ and that in up to $40 \%$ of cases a second EBP is required. ${ }^{5,6}$ They should be made aware that the time course of symptoms and for treatment is frequently prolonged.

Irrespective of these imperfect outcomes, few would argue that EBP should be discussed with women otherwise incapacitated by PDPH, given that no other treatments offer the hope of rapid permanent relief, good initial symptomatic relief, or the correction of hearing loss. It has been recommended that EBP be performed sooner rather than later, ${ }^{7}$ on the basis that unresolved PDPH is, rarely, associated with sequelae such as seizures, cranial subdural hematoma and 'coning'. On the other hand, it is never too late, because successful EBP is described after PDPH of more than a year in duration.

\section{"When, and how, should EBP be performed?}

The historical trail leading to facets of current practice makes interesting reading. ${ }^{8}$ Much is written on the best volume of autologous blood for EBP, but practice varies widely, with renowned obstetric anesthesiologists adamant that 10,20 and $30 \mathrm{~mL}$ volumes are optimal! I recently looked after a patient who had received a $2 \mathrm{~mL}$ EBP from a radiologist (it didn't work!). This specialist clearly supported Gormley's approach from 1960, when he described 100\% success from 2 to $3 \mathrm{~mL}$ of autologous blood (including relief of his own post-myelogram headache!). ${ }^{2}$ In the 1970 s 5 to $10 \mathrm{~mL}$ became popular, with permanent relief claimed in $90 \%$ of patients. ${ }^{9}$ Such volumes increase subarachnoid pressure significantly, ${ }^{10}$ and the tamponade and displacement of cerebrospinal fluid (CSF) cephalad, as well as the "clot-plug", have been proposed as reasons for successful patch. Crawford was the first to note a substantial rate of headache recurrence and claimed better success with $20 \mathrm{~mL}$. ${ }^{11}$ This became the recommended target volume, although some failed to find better efficacy as the volume increased. ${ }^{4,5}$ The first imaging studies of EBP showed wide spinal segmental distribution of $15 \mathrm{~mL}$ of blood. $^{12}$ Recent retrospective or non-randomized series have suggested optimal results with up to 30 $\mathrm{mL}$, or the maximum tolerated volume, ${ }^{13,14}$ the disadvantage being that backache is more frequent. ${ }^{4}$ So, the jury is out, and, in the absence of a randomized trial, there is no convincing evidence for any particular volume above about $10 \mathrm{~mL}$.

The efficacy of EBP appears to vary, not only with the severity of symptoms, but also according to its timing in relation to dural puncture. Prospective observational data suggests EBP performed a week after dural puncture, compared with within $24 \mathrm{hr}$, is more likely to be successful.

So, should we act pre-emptively? The traditional approach was to resite the epidural catheter, providing an opportunity to deliver a prophylactic EBP (PEBP) prior to the onset of headache. A number of retrospective and small prospective studies support PEBP and some reviewers recommend it. ${ }^{15}$ After PEBP, headache rates vary widely, from only $5-20 \%^{1}$ to $40-80 \% 5,6,16$ compared with $75-90 \%$ if no action is taken. So why do other reviewers disagree, ${ }^{17}$ and why do the majority of academic units in North America, UK and Europe not routinely offer PEBP? The popularity of PEBP has fallen further, a very recent survey from the UK finding it used in only $3 \%$ of units. ${ }^{18}$ Level 2 evidence from randomized trials is at last emerging. Two studies evaluated 65 obstetric patients and indicate modest efficacy, but lower success than therapeutic EBP. ${ }^{1}$ The best and most recent trial only included dural puncture with a 17-gauge Tuohy needle, used a sham injection in the control group, $20 \mathrm{~mL}$ of blood, blinded observers and lengthy follow-up. ${ }^{17}$ This trial found a similar incidence (approximately $60 \%$ ), similar severity but a shorter duration of PDPH in those who had received PEBP. Therapeutic EBP was required by about $50 \%$ of each group, which is consistent with recent observational series. ${ }^{5,6}$

Given this knowledge, I do not believe PEBP is justified. Between 10 and 40\% will not experience significant PDPH anyway and a third to a half of those that do will not need a therapeutic EBP. Half those having PEBP will require another (therapeutic) EBP.

The decision is one of risk benefit, and informed consent should be obtained. An EBP has side effects and complications. The catheter tip may be paravertebral, raising theoretical concern about the mass effect on nerve roots; and if located in the subarachnoid space, injection of a large volume of blood is clearly undesirable. I believe the option of conservative ther- 
apy as the first line modality of care should be provided. Given the increasing popularity of intrathecal insertion of the epidural catheter (rather than epidural re-insertion), ${ }^{18}$ which also appears to reduce the incidence and severity of PDPH,${ }^{19}$ the utility of PEBP has been further reduced.

When PDPH occurs, the optimum timing of intervention is also in dispute. The oft-quoted randomized trial on this subject found a dramatic difference in headache rate when EBP was performed before or after $24 \mathrm{hr}$, but many of those in the delayed group had headache after spinal needle, rather than epidural needle, puncture and no detail was provided..$^{20} \mathrm{~A}$ number of observational series also support delaying therapeutic EBP for 24 to $48 \mathrm{hr}$ or more. This appears to make sense, as symptoms may have begun to resolve anyway, a fibroblastic response at the site of puncture is present within $24 \mathrm{hr}$, and the effect of local anesthetic has dissipated. Some injected blood collects on the inner surface of the duro-arachnoid membrane and CSF is procoagulant, promoting the "clot-patch", but lidocaine and to a lesser extent bupivacaine and ropivacaine interfere with coagulation by inhibition of platelet aggregation and granule release. EBP within four days of dural puncture is an independent risk factor for failure of $\mathrm{EBP} ;{ }^{4}$ the longer the delay before EBP the more likely it is to prove successful $^{4,13,14}$ and when performed within $48 \mathrm{hr}$ a higher rate of repeat EBP is reported. ${ }^{5,14}$ In my audit of 58 therapeutic EBP, only seven were performed within $24 \mathrm{hr}$. Six were successful, but five women subsequently experienced recurrent headache (overall rate $30 \%) .{ }^{5}$ In the absence of data from randomized trials and faced with a parturient with an incapacitating headache (30-40\% of PDPH present within $24 \mathrm{hr}$ ), 5,6 it is reasonable to argue that early EBP should be considered, ${ }^{7,15}$ especially if aggressive symptomatic therapy is used as a temporizing measure for $48 \mathrm{hr}$.

\section{"EPB is very safe"}

Most obstetric anesthetists would contend that EBP is "very safe". There are certainly a number of small series, involving a few hundred patients in total, which have not reported serious complications. It concerns me a little, however, that many appear unaware of several very serious complications reported in association with EBP and that I often encounter patients to whom no risks have been disclosed. Quantifying the risk is a problem, because data are insufficient. No single unit is likely to gather enough cases and statistically, the absence of a particular complication after 1,000 cases still gives the upper limit of risk using a onesided $95 \%$ confidence interval of 3 in 1,000 . If one of the many serious complications (cranial subdural hematoma, abducens or facial nerve paresis, cauda equina syndrome, a seizure, aseptic meningitis or arachnoiditis), ${ }^{2}$ fell within this incidence range, I think many patients would consider this significant.

Transient bradycardia is a side effect of EBP in both animal models and patients and may reflect changes in spinal pressure (which are limited by slow injection) or autonomic changes with patient positioning. This and the mild febrile reactions also reported are unlikely to cause concern, although the latter might initiate investigation for an infective complication. The most common side effect of EBP is immediate or late back pain. Mild pain during injection is very common once more than 10 to $12 \mathrm{~mL}$ of blood is injected and most patients receiving 15 to $25 \mathrm{~mL}$ report some pain, although the pressure (and volume) at which this occurs varies widely. Significant pain sometimes limits the volume administered, in my experience particularly when repeat EBP is performed, and this may reflect high epidural or subarachnoid pressure and/or nerve root irritation or compression. ${ }^{21}$ Mild, self-limiting back pain following EBP is also common $(35-70 \%)^{5,18,33}$ and may result from tracking of blood into the intervertebral ligaments and subcutaneous fat. ${ }^{21}$ Surveys show that many centres have experience with patients who suffered persistent back (and much less commonly neck) pain; symptoms of meningeal irritation; or infection. Severe persisting radicular pain mandates exclusion of spinal subdural or subarachnoid hematoma. Passage of blood into the subarachnoid space during EBP could cause aseptic meningitis or late arachnoiditis, but is very unlikely unless a large volume is injected intrathecally. A recent report of paraplegia and cauda equina syndrome after EBP is sobering ${ }^{22}$ and reiterates the point that EBP is an invasive procedure that is not completely benign. Serious complications such as epidural infection and meningitis emphasise the importance of a strict aseptic technique. Until a large, accurate, prospective, multicentre database has been established, we are not in a position to accurately estimate risks associated with EBP.

And should we perform repeated EBP (once or more)? There are serious complications of dural puncture that might be worsened by EBP (e.g., cranial subdural hematoma), and various other postpartum or pathological headache (e.g., from tumour or cortical vein thrombosis) can mimic PDPH, with postural elements. Before every EBP, a diagnostic sieve must be applied and when in doubt, appropriate investigation and imaging performed. 
"EBP stops headache by plugging the hole"

This traditional concept of a "clot-plug", first espoused by Sicard in 1902, dictates that stopping CSF leak corrects the low CSF pressure and relieves downward traction on pain-sensitive cranial structures (venous sinuses and intracranial nerves). This explanation has elements of fact but fails to account for features such as the immediate relief of headache; the poor relationship between headache and CSF pressure or volume; and the failure of magnetic resonance imaging to demonstrate structural sagging. ${ }^{23}$ The "mass-effect" postulate states that EBP increases epidural and subarachnoid pressures significantly, reducing epidural distensibility, shifting CSF cephalad to increase CSF volume. ${ }^{8}$ In contrast to transient increases seen with injection of saline or Dextran, the pressure rise after blood is sustained ${ }^{24}$ and the mass effect is visible for several hours. ${ }^{21}$ On the basis of the "clot-plug" and "mass effect" theories, we have all learnt to inject the autologous blood at or below the level of the dural puncture. Is this valid? - no studies have addressed whether this makes any difference.

Cerebral vasodilatation occurs in response to dural puncture, in accordance with the Monro-Kellie hypothesis regarding maintenance of intracranial volume. This may be due to activation of adenosine receptors, following a fall in cranial CSF volume because of a caudad shift in differential pressure. Consequently PDPH has features characteristic of other vascular headaches. In the erect position, lumbar subarachnoid space compliance increases in the presence of anatomical joining of the subarachnoid and epidural space, lowering cranial CSF pressure further. Unless the increase in compliance is low (as in the very young and the elderly), the consequence of relative intracranial hypotension is compensatory venodilation and possibly arterial vasodilation. ${ }^{25}$ EBP reverses vasodilatation and cerebral blood flow falls immediately, possibly mediated by deactivation of adenosine receptor responses as intraspinal pressures rise, or by release of vasoactive substances from the dura (subdural blood is a powerful stimulant of cerebral vasoconstriction). Another untested theory is that EBP blocks the normal lymphatic and arachnoid villae drainage of CSF from the subarachnoid space into the venous system, allowing rapid replenishment of CSF volume. A further postulate is that subdural injection of small volumes of blood will be effective, given success with this approach after failed EBP ${ }^{8}$

It seems reasonable to assume that PDPH is complex, has several contributory mechanisms, with interindividual variability, and consequently EBP is not a panacea. Headache caused by intracranial air, for example, is unlikely to respond to EBP. Achieving cerebral vasoconstriction by pharmacological means is appealing, but results to date from treatment with drugs such as caffeine, theophylline, sumatriptan and adrenocorticotrophic hormone are disappointing. The most effective option is EBP, but let's dispel the myths and search for more answers!

\section{References}

I Sudlow C, Warlow C. Epidural blood patching for preventing and treating post-dural puncture headache (Cochrane Review). In: The Cochrane Library, Issue 4, 2003. Chichester, UK. John Wiley \& Sons Ltd.

2 Gormley JB. Treatment of postspinal headache. Anesthesiology 1960; 21: 565-6.

3 Bart AJ, Wheeler AS. Comparison of epidural saline placement and epidural blood placement in the treatment of post-lumbar-puncture headache.

Anesthesiology 1978; 48: 221-3.

4 Safa-Tisseront V, Thormann F, Malassine P, et al. Effectiveness of epidural blood patch in the management of post-dural puncture headache. Anesthesiology 2001; 95: 334-49.

5 Banks S, Paech M, Gurrin L. An audit of epidural blood patch after accidental dural puncture with a Tuohy needle in obstetric patients. International Journal of Obstetric Anesthesia 2001; 10: 172-6.

6 Williams EJ, Beaulieu P, Fawcett WJ, Jenkins JG. Efficacy of epidural blood patch in the obstetric population. International Journal of Obstetric Anesthesia 1999; 8: 105-9.

7 Reynolds F. Dural puncture and headache. Avoid the first but treat the second. BMJ 1993; 306: 874-6.

8 Harrington BE. Postdural puncture headache and the development of the epidural blood patch. Reg Anesth Pain Med 2004; 29: 136-63.

9 DiGiovanni AJ, Dunbar BS. Epidural injections of autologous blood for postlumbar puncture headache. Anesth Analg 1970; 49: 268-71.

10 Coombs DW, Hooper D. Subarachnoid pressure with epidural blood patch. Reg Anesth 1979; 4: 3-6.

11 Crawford JS. Epidural blood patch (Letter). Anaesthesia 1985; 40: 381.

12 Szeinfeld M, Ibmeidan I, Moser MM, et al. Epidural blood patch: evaluation of the volume and spread of blood injected into the epidural space. Anesthesiology 1986; 64: 820-2.

13 Ascanio RS, Evans RE, Siegle J, Curry CS. Successful therapeutic epidural blood patch in obstetrics: later rather than sooner. Anesthesiology 1999; 92: 96 (abstract).

14 Rutter S, Russell R, Popat M. Efficacy of epidural blood patch in the obstetric population (Letter). International 
Journal of Obstetric Anesthesia 2000; 9: 69.

15 Duffy PJ, Crosby ET. The epidural blood patch. Resolving the controversies. Can J Anesth 1999; 46; 878-86.

16 Scavone B, Wong CA, Sullivan JT, Yaghmour E, Sherwani S, McCarthy RJ. Efficacy of prophylactic epidural blood patch in preventing post dural puncture headache in parturients after inadvertent dural puncture. Anesthesiology 2004 Dec; 101 (6): 1422-7.

17 Vasdev GM, Southern PA. Postdural puncture headache: the role of epidural blood patch. Current Pain and Headache Reports 2001; 5: 281-3.

18 Baraz $R$, Collis RE. A survey of the management of accidental dural puncture and post-dural puncture headache in UK obstetric practice (abstract).

International Journal of Obstetric Anesthesia 2004; 13: S15-O24.

19 Ayad S, Demian Y, Narouze SN, Tetzlaff JE. Subarachnoid catheter placement after wet tap for analgesia in labor: influence on the risk of headache in obstetric patients. Reg Anesth Pain Med 2003; 28 : 512-5.

20 Loeser EA, Hill GE, Bennett GM, Sederberg JH. Time vs. success rate for epidural blood patch. Anesthesiology 1978; 49: 147-8.

21 Beards SC, Jackson A, Griffiths AG, Horsman EL. Magnetic resonance imaging of extradural blood patches: appearances from $30 \mathrm{~min}$ to $18 \mathrm{~h}$. Br J Anaesth 1993; 71: 182-8.

22 Diaz JH. Permanent paraperesis and cauda equina syndrome after epidural blood patch for post-dural-puncture headache. Anesthesiology 2002; 96: 1515-7.

23 Grant R, Condon B, Hart I, Teasdale GM. Changes in intracranial CSF volume after lumbar puncture and their relationship to post-LP headache. J Neurol Neurosurg Psychiatr 1991; 54: 440-2.

24 Kroin JS, Nagalla SKS, Buvanendran A, et al. The mechanisms of intracranial pressure modulation by epidural blood and other injectates in a postdural puncture rat model. Anesth Analg 2002; 95: 423-9.

25 Levine DN, Rapalino O. The pathophysiology of lumbar puncture headache. J Neurolog Sci 2001; 192:

1-8. 